\title{
Imaging in Ovarian Dermoid Cyst- Floating Ball: A Pathognomonic Sign of Mature Cystic Teratoma
}

\author{
ANITHA KINI', ANIL KUMAR SHUKLA², NIKITHA REDDY ${ }^{3}$
}

\section{ABSTRACT}

Teratomas in the ovaries can be classified as Mature Cystic Teratomas (MCT), immature teratomas and mono-dermal teratomas which includes Struma ovarii, neural tumours and carcinoids. Multimodality imaging like Ultrasonography (US), Computed Tomography (CT) and Magnetic Resonance Imaging (MRI) approach can be used in the correct diagnosis of mature cystic ovarian teratomas. On US, it is characterised by echogenic sebaceous material and calcification. Fat attenuation can be visualised on CT. The sebaceous component is better identified with MRI fat suppression techniques. Immature teratomas are heterogeneous, partly solid lesions with scattered or coarse calcifications and small foci of fat. The imaging features of Struma ovarii is non-specific. We present a case of MCT which has a pathognomonic appearance of globular ball floating in a cyst with fat-fluid level.

\section{CASE REPORT}

A 20-year-old female patient came with complains of cramping type of pain in the abdomen since four months aggravating in the night, non-radiating, not relieved on medications. She also complains of white discharge per vagina since one month, scanty in amount. No itching, no bladder or bowel disturbances, no menstrual disturbances, no history of fever, vomiting or burning micturition.

On per abdomen examination, a midline mass corresponding to 20 weeks size was seen which was hard in consistency. On per vaginal examination, uterus was 20 weeks size, ante-verted. Right side fornix fullness was present. Left fornix was obliterated.

Laboratory investigations revealed mildly elevated white blood cell count $(14,000 / \mathrm{mL}$ of blood), rest of the haemogram, liver and renal function tests were within normal limits. Tumour markers CA 19-9, CEA (Carcino-embryonic antigen), CA 125, AFP (alpha feto protein) were within normal limits.

The patient was then referred to Department of Radio-diagnosis for ultrasound and Contrast Enhanced Computed Tomography (CECT) of abdomen and pelvis for further evaluation.

On ultrasound examination, there was a large thick walled cystic lesion with multiple well-defined hyperechoic areas measuring $15.7 \times 13.5 \times 14.4 \mathrm{~cm}$ extending from left adnexa to epigastric region [Table/Fig-1]. The patient was made to lie in left lateral decubitus which showed movement of globular hyperechoic areas within the cyst.

On Non-Contrast CT (NCCT), there was a well-defined, midline, cystic lesion with multiple globular fat densities measuring $10 \times 14 \times 17 \mathrm{~cm}$ with extension into left adnexa. Tiny calcific foci noted in anterior portion. Fat planes between urinary bladder and uterus was maintained. Lesion extends superiorly up to the $L 4$ vertebra [Table/Fig-2]. On post contrast images, there was no enhancement of the lesion [Table/Fig-3]. Floating fat balls when present are considered pathognomonic as noted in our case. MRI was not performed as the patient had crampy abdominal pain. The patient underwent left oophorectomy and with complete excision of the cyst. Post-operative period was uneventful.

On histopathological examination it revealed mature cystic teratoma with no malignant transformation.
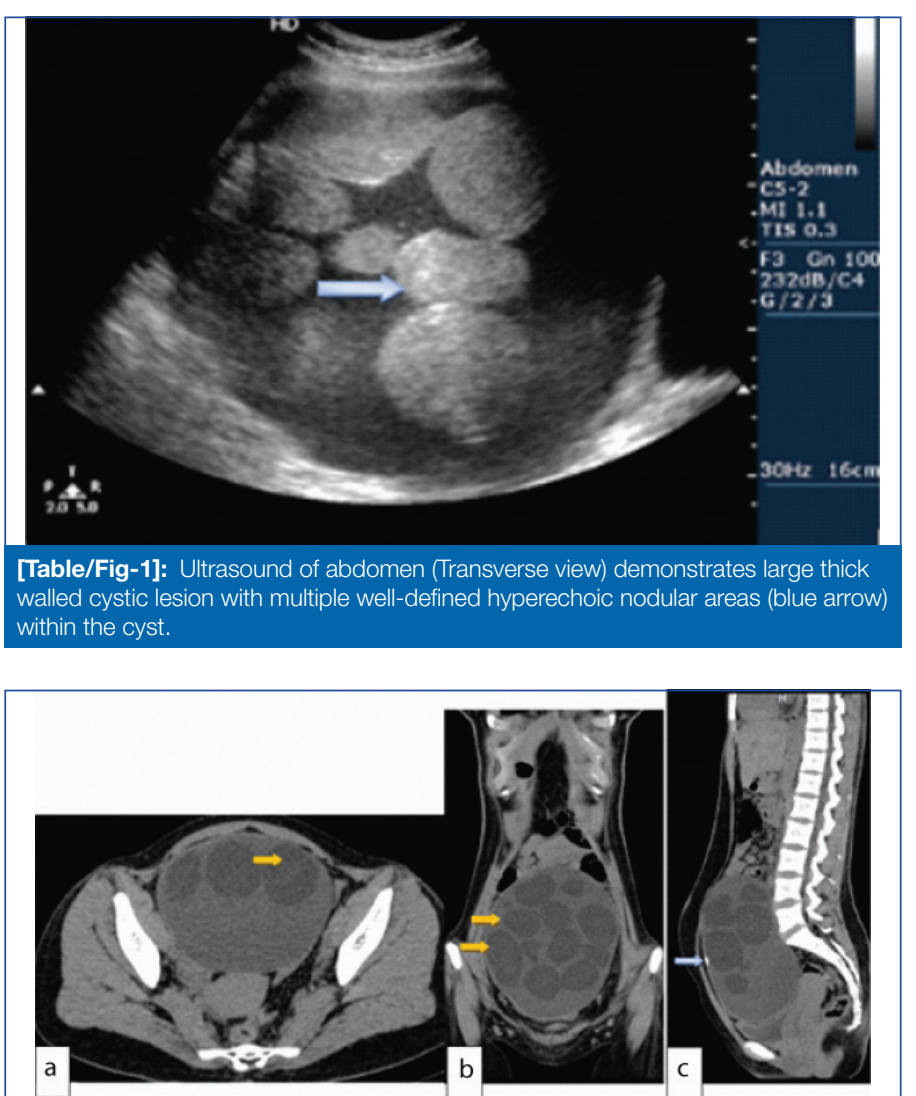

[Table/Fig-2 (a-c)]: Axial, coronal and sagittal NCCT images demonstrates a welldefined, midline, cystic lesion with multiple globular fat densities (brown arrows) with extension into left adnexa. Tiny calcific foci noted in anterior portion (blue arrow). Fat planes between urinary bladder and uterus was maintained. Lesion extends superiorly up to the $L 4$ vertebra.

\section{DISCUSSION}

Mature Cystic Teratoma (MCT), also termed as dermoid cyst is the most common benign ovarian tumour, $50 \%$ of those are present in paediatric age group [1-6]. As they are slow growing tumour, they are usually asymptomatic [4-6]. They are rarely diagnosed before puberty and its growth ceases at menopause [6-9]. Typically, they contain derivatives of the three germ layers, the ectoderm, the mesoderm and the endoderm [7]. The diversity of tissues in teratomas results in a wide variety of characteristics in imaging studies. 


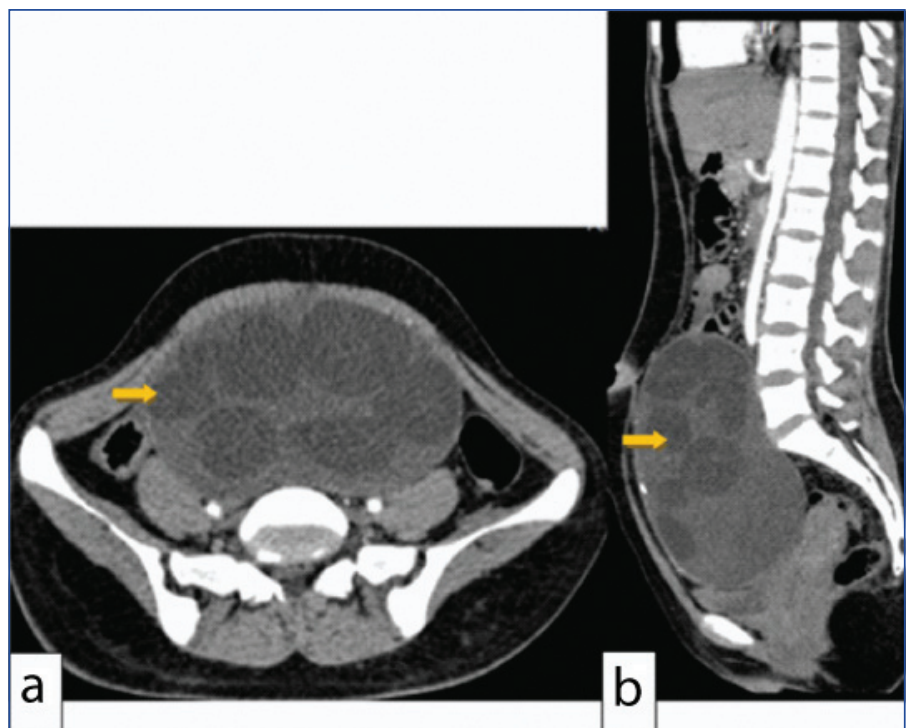

[Table/Fig-3 (a,b)]: Axial and sagittal CECT images demonstrates a non-enhancing well defined, midline, cystic lesion with multiple globular fat densities (brown arrows) with extension into left adnexa. Fat planes between urinary bladder and uterus is maintained. Lesion extends superiorly up to the $L 4$ vertebra.

Imaging features are unilateral, unilocular, smooth-surfaced lesions, to $15 \mathrm{~cm}$ in size. They may contain sebaceous material or fat, hair, bone or calcification, thyroid tissue, and bronchial tissue. Intracystic fat can be seen as solid when epithelial debris and hair form conglomerates [1,4]. In a large series reviewing 370 cases of benign ovarian teratomas, Rha SE et al., reported that a fluid-fluid level within a cystic ovarian tumour may strongly suggest MCT, but it is pathognomonic for a MCT when there is a floating nodule observed within the lesion in any of the imaging modalities [4].

In our case, as a floating ball within the lesion was observed, a diagnosis of MCT was made, and laparotomy with left oophorectomy confirmed the diagnosis. Our case shows an unusual coexistence of floating fat ball appearance with fat-fluid level which has not been reported.

Other cystic ovarian tumours including necrotic material or blood, endometrioma or haemorrhagic ovarian cysts may also include blood elements at varying degeneration stages, and they may show a fluid-fluid level; thus, it may not always be a sufficient finding for the diagnosis of cystic ovarian teratomas $[1,8,9]$. It has been reported that level or floating nodule appearance are not seen in non-teratomatous masses [1].

Based on the configuration and components within the ovarian cystic teratomas they are classified into 3 types:

- Layering secondary to floating debris within the tumour is seen.

- $\quad$ Cyst contains nodular or mural protrusions.

- $\quad$ Cyst contains a fat fluid level [9].

Our case has the unusual classic appearance of both fat-fluid level and floating ball. Floating nodules within the cystic ovarian teratomas include fat, hair, and soft tissue. Floating nodules consisting of a mixture of fat and hair are seen as hyperechoic with ultrasonography and produce intense posterior acoustic shadowing. In our case, the floating nodule had the same property. An anechoic cystic component represents pure sebum that is liquid at normal body temperature [5]. US examination is usually enough for demonstrating a floating fat ball $[5,6]$, US may not be sufficient for accurate diagnosis when a floating ball produces strong acoustic shadowing, as it was in our case.

In this situation, CT may be more beneficial than US for the evaluation of the inner texture of the lesion. Moreover, CT examination at the decubitus position may be helpful in showing mobility of the fat ball. In patient with a cystic ovarian mass, fat-fluid level, fluid-fluid level with the presence of a fatty component and a floating nodule appearance on CT, is accepted pathognomonic for MCT $[1,10,11]$. $\mathrm{MRI}$, with a high degree of soft tissue resolution and multi-planar imaging properties, has become the imaging modality of choice for examining the pelvis, especially in women of reproductive age, in order to avoid ionizing radiation. T1 weighted MRI gives diagnostic information for lesions. On T1weighted MRI, most cystic teratomas show hyperintensity since they include high fat tissue content. Calcifications, bone, hair, and fibrous tissue that are usually seen in teratomas are observed as hypointense areas [11]. In a patient with an ovarian mass, a fat fluid level or a fluid-fluid level in the presence of a fatty component, and floating ball appearances on CT or MRI is diagnostic of cystic teratomas. Additionally, a few CT slices in lateral decubitus position, the mobility of floating ball can be demonstrated to confirm the diagnosis.

\section{CONCLUSION}

Imaging plays a vital role in the diagnosis and characterisation of ovarian teratomas. It can also predict whether there is presence of any malignant transformation. Our case has the unusual appearance of both fat-fluid level and floating ball, which is considered as pathognomonic feature of MCT of ovary.

\section{REFERENCES}

[1] Hsiang CW, Liu WC, Huang GS, Hsu HH, Chang WC. A floating ball: A pathognomonic sign of ovarian cystic teratoma. QJM. 2014;107(4):319-20.

[2] Sahin H, Abdullazade S, Sanci M. Mature cystic teratoma of the ovary: A cutting edge overview on imaging features. Insights into Imaging. 2017;8(2):227-41.

[3] Sahin H, Akdoğan Al, Ayaz D, Karadeniz T, Sancı M. Utility of the "floating ball sign" in diagnosis of ovarian cystic teratoma. Journal of Turkish Society of Obstetrics and Gynecology. 2019;16(2):118-23.

[4] Rha SE, Byun JY, Jung SE, Kim HL, Oh SN, Kim H, et al. Atypical CT and MRI manifestations of mature ovarian cystic teratomas. American Journal of Roentgenology. 2004;183(3):743-50.

[5] Tandon A, Agarwal R, Tandon R, Prakash M. Multiple intracystic floating balls: An unusual but unique sonographic pattern of mature cystic teratomas. Case Reports. 2011;2011(jun30 1):bcr0320113962-bcr0320113962.

[6] Park SB, Kim JK, Kim KR, Cho KS. Imaging findings of complications and unusual manifestations of ovarian teratomas. Radiographics. 2008;28(4):969-83.

[7] Gürel H, Gürel SA. Ovarian cystic teratomas with a pathognomonic appearance of multiple floating balls: A case report and investigation of common characteristics of the cases in the literature. Fertility and Sterility. 2008;90(5):2008.e17-2008.e19.

[8] Outwater EK, Siegelman ES, Hunt JL. Ovarian teratomas: Tumour types and imaging characteristics. Radiographics. 2001;21(2):475-90.

[9] Yazıcı B, Erdoğmuș B. Floating ball appearance in ovarian cystic teratomas. Diagn Interv Radiol. 2006;12:136-38.

[10] Chang-Chien CY, Tzeng WS, Shu GHF, Mak CW. Cystic ovarian teratomas with floating globules. J Radiol Sci. 2012;37:123-26.

[11] Øwre A, Pedersen JF. Characteristic fat-fluid level at ultrasonography of ovarian dermoid cyst. Acta Radiologica. 1991;32(4):317-19.

\section{PARTICULARS OF CONTRIBUTORS:}

1. Senior Resident, Department of Radiology, Raja Rajeswari Medical College and Hospital, Bengaluru, Karnataka, India.

2. Professor, Department of Radiology, Raja Rajeswari Medical College and Hospital, Bengaluru, Karnataka, India.

3. Postgraduate Resident, Department of Radiology, Raja Rajeswari Medical College and Hospital, Bengaluru, Karnataka, India.

NAME, ADDRESS, E-MAIL ID OF THE CORRESPONDING AUTHOR:

Dr. Anitha Kini,

\#3/1, $9^{\text {th }}$ Main Road, Banashankari $2^{\text {nd }}$ Stage, Bengaluru, Karnataka, India.

E-mail: anusai1709@gmail.com

Date of Submission: Jul 15, 2019 Date of Peer Review: Jul 31, 2019 Date of Acceptance: Aug 20, 2019 Date of Publishing: Oct 01, 2019 